\section{Chronisch-entzündliche Darmerkrankungen}

\section{Dickdarmkarzinomrisiko und Überwachungsstrategien}

Inflammatory bowel diseases.

\section{Risk of colorectal cancer and surveillance strategies}

\section{H. Scherübl}

B. M. Wittig

J. C. Hoffmann

M. Zeitz
Patienten mit langjähriger chronisch-entzündlicher Darmerkrankung (CED) und Kolonbefall haben ein erhöhtes Risiko für das kolorektale Karzinom (KRK). In dieser Arbeit werden Empfehlungen zur endoskopischen Überwachung von Patienten mit CED gegeben und unter Berücksichtigung der aktuellen Literatur diskutiert.

\section{Erhöhtes Risiko füir KRIK}

Obwohl lediglich 1-2\% der Erkrankungen an einem KRK auf eine CED zurückzuführen sind, bedeutet es für die Patienten mit langjähriger Colitis ulcerosa (CU) oder Crohn-Colitis ein ernstzunehmendes Risiko und ist für bis zu 1/6 der Todesfälle bei CED verantwortlich $(2,3,14)$. Das Risiko zur Entstehung eines KRK bei CU steigt eindeutig mit Erkrankungsdauer, Ausdehnung der Entzündung sowie frühem Krankheitsbeginn. Nach einer Metaanalyse (116 Studien) beträgt die Prävalenz des KRK bei CU-Patienten 3,7\% (3). In dieser Studie lagen die KRK-Inzidenzraten bei 2,1\% nach einer Erkrankungsdauer von 10 Jahren, bei 8,5\% nach 20 und bei 17,8\% nach 30 Jahren. Neben der Dauer ist die Ausdehnung der CU einen eindeutigen Risikofaktor für die Entstehung eines KRK. Patienten mit reinem Befall des Rektums erkranken deutlich seltener als Patienten mit einer Pancolitis ulcerosa an einem KRK. Darüber hinaus steigt das Risiko für das KRK signifikant bei Vorliegen einer primär sklerosierenden Cholangitis, wobei es dann häufig im proximalen Kolon liegt und eine deutlich ungünstigere Prognose aufweist. Das kumulative Dysplasie- und Karzinomrisiko ist bei diesen Patienten nach 30 Jahren um einen Faktor 5-10 gegenüber Patienten mit einer alleinigen CU erhöht (8). Eine Risikoerhöhung wird dabei nicht nur direkt für das KRK, sondern bereits für das Vorliegen einer Dysplasie oder DNAAneuploidie beobachtet. Ein Überwachungsprogramm sollte insbesondere die Patienten mit erhöhtem Risiko einschließen (Tab.1).
Tab.1 Risikofaktoren für die Karzinomentstehung bei CED.

\begin{tabular}{ll}
\hline Risikofaktor & RR \\
\hline Colitis ulcerosa & \\
Befallsmuster: & \\
- Proktitis & 1,7 \\
- Linksseiten-Colitis & 2,8 \\
- Pancolitis & 14,8 \\
Langjährige Colitis (> 8 Jahre) & $5-19$ \\
- 10 jahre & 2,1 \\
- 20 jahre & 8,5 \\
- 30 jahre & 17,8 \\
Primär sklerosierende Cholangitis & $9-18$ \\
Positive Familienanamnese für KRK & 2,3 \\
Morbus Crohn & \\
Colitis Crohn & $1-5,6$ \\
RR=relatives Risiko. KRK=kolorektales Karzinom. CED=chronisch entzündliche \\
Darmerkrankungen
\end{tabular}

Patienten mit Crohn-Colitis scheinen ein erhöhtes Risiko für das Auftreten eines KRK zu haben, sofern die Krankheitsdauer und das Ausmaß des Kolonbefalls berücksichtigt wird. Ekbom et al. (4) beschreiben bei 1655 Patienten mit Morbus Crohn ein 2,5-fach erhöhtes Risiko, bezogen auf die Patienten mit Kolonbefall sogar ein 5,6fach erhöhtes Risiko. Bei Ileozökalbefall betrug das relative Risiko 3,2, bei reinem Dünndarmbefall war das Risiko für das KRK nicht erhöht (4). Das Risiko für das Kolonkarzinom bei Morbus Crohn steigt mit der Dauer der Erkrankung und dem Ausmaß des Kolonbefalls, weist einen vergleichbaren Verlauf und Prognose wie bei der CU auf und geht ebenfalls aus intraepithelialen Neoplasien (IEN) hervor (2). Im Gegensatz zum sporadischen oder CU-assoziierten Karzinom tritt jedoch das Kolonkarzinom bei Morbus Crohn, entsprechend des entzündlichen Befalls, vor allem proximal auf (2). Drei größere Studien weisen bei langjährigem Morbus Crohn, allerdings ohne extensiven Kolonbefall, kein erhöhtes Risiko für das KRK auf, zeigen je-

Institut

Charité - Universitätsmedizin Berlin, Campus Benjamin Franklin, Medizinische Klinik I - Gastroenterologie,

Infektiologie und Rheumatologie

Korrespondenz

Priv.-Doz. Dr. med. Bianca Wittig - Medizinische Klinik 1, Charité - Universitätsmedizin Berlin

Campus Benjamin Franklin · Hindenburgdamm 30 · 12200 Berlin · Tel.: 030/84453534

· Fax: 030/84454481 · E-Mail: bianca.wittig@charite.de

eingereicht: $17.5 .2004 \cdot$ alkzeptiert: 9.8.2004

Bibliografie

DOI: $10.1055 / \mathrm{s}-2004-831385$

Dtsch Med Wochenschr 2004;129:S96-S98 - ㄷ Georg Thieme Verlag Stuttgart · New York · ISSN 0012-0472 
doch ein 15-60fach erhöhtes Risiko für die Entstehung des insgesamt sehr seltenen Dünndarmkarzinoms (6).

kurzgefasst: Bei langjähriger Colitis ulcerosa und Morbus Crohn mit Kolonbefall (Crohn-Colitis) steigt das Risiko für ein KRK mit Dauer der Erkrankung und Ausdehnung der Entzüindung.

\section{Dysplasie-Karzinom-Sequenz des Colitis-assoziierten IKRK}

Nach heutigem Wissen entsteht das Karzinom bei CED aus intraepithelialen Neoplasien (früher Dysplasien). Molekulargenetisch sind die klassischen Veränderungen beim sporadischen KRK wie Mutationen des APC- oder DCC-Gens beim Colitis-assoziierten Karzinom deutlich seltener nachweisbar, wogegen sich in bis zu 50\% der Colitis-assoziierten Karzinome K-ras- und p53-Mutationen finden. Bisher gibt es keine Marker, die routinemäßig in der breiten Untersuchung von Risikopatienten eingesetzt werden könnten, Mutationsnachweise in abgeschilferten Epithelien aus dem Stuhl sind aber prinzipiell möglich.

\section{Überwachungsstrategie zur Früherkennung von Colitis- assoziierten Neoplasien}

In retrospektiven Studien wurde gezeigt, dass die koloskopische Überwachung mit multiplen Biopsieentnahmen zu einer Reduktion der KRK-Mortalität durch vermehrten Nachweis von IEN und Karzinomen in frühen Stadien führt. Da die Prognose des KRK in erster Linie vom Tumorstadium bei Diagnose abhängt, wird allen Patienten mit einem über 8-jährigen Verlauf einer (sub)totalen CU eine jährliche endoskopische Überwachung (im Remissionsstadium) empfohlen (8). Zu diesem Zeitpunkt beträgt das Krebsrisiko etwa 3\%. Auch jugendliche Patienten mit ausgedehnter Colitis sollten nach 8-jährigem Verlauf in ein Überwachungsprogramm eingeschlossen werden, unklar ist jedoch, ob dies auch bei lediglich distalem Befall zu gelten hat $(8,10)$. Da IEN und Karzinome bei der linksseitigen Colitis erst später auftreten, sollte hier nach 15-jährigem Krankheitsverlauf (im Remissionsstadium) eine jährliche Koloskopie mit Biopsien erfolgen. Überwachungs-Koloskopien sollten unbedingt im entzündungsfreien Intervall erfolgen, da entzündliche Veränderungen häufig sehr schlecht von intraepithelialen Neoplasien (IEN) zu unterscheiden sind.

Der Nachweis einer IEN gilt als Goldstandard, um Risikopatienten zu identifizieren. Die IEN ist als eine Neoplasie des Epithels definiert. Einschränkend muss jedoch bemerkt werden, dass die Identifikation einer IEN in flacher, nicht-polypoider Schleimhaut sehr von der Erfahrung des beurteilenden Pathologen abhängig ist und die Klassifikation einer erheblichen inter- und intraindividuellen Variabilität unterworfen ist. Die Anzahl der entnommenen Biopsien bei jeder Überwachungskoloskopie ist ein entscheidender Faktor in der Detektion von dysplastischen oder karzinomatösen Veränderungen. Aus einer Untersuchung an Resektaten lässt sich ableiten, dass bei Entnahme von $33 \mathrm{Bi}-$ opsien pro Koloskopie dysplastische Veränderungen zu 90\% nachgewiesen werden können. Bei Entnahme von 56 Biopsien steigt die Sensitivität auf 95\%. In einer anderen Studie (13) wurden unter Imitation der endoskopischen Biopsieentnahme bei 100 Kolektomiepräparaten (von Patienten mit einem Kolonkarzinom bzw. mit einer hochgradigen Dysplasie) jeweils 4 Biopsi- en aus 8 unterschiedlichen Lokalisationen entnommen. Bei 26\% der Kolektomiepräparate mit einem Karzinom konnte keine Dysplasie nachgewiesen werden. Somit lässt sich die Sensitivität und Spezifität mit jeweils 74\% für die Detektion eines Karzinoms berechnen. Aufgrund der vorliegenden Datenlage wird somit empfohlen, je 4 Kolonbiopsien in $10 \mathrm{~cm}$ Abständen aus allen Kolonabschnitten, d.h. insgesamt 40-50 Biopsien zu entnehmen. Zudem wird jedes suspekte Areal separat biopsiert.

Entscheidend für die Prognose der Patienten mit CU ist natürlich das gezielte Auffinden prämaligner Schleimhautabschnitte. Das Erkennen von Colitis-assoziierten neoplastischen Schleimhautveränderungen während der Koloskopie ist schwierig, da diese Veränderungen auch in makroskopisch unauffälliger Darmschleimhaut gefunden werden können. Vielversprechende Ergebnisse wurden in den letzten Jahren zu verfeinerten endoskopischen Untersuchungsmethoden (Chromo-, Fluoreszenz- und Zoom-Endoskopie) vorgestellt (7). Diese diagnostischen Verfahren haben bislang noch keinen gesicherten Stellenwert in der Routinediagnostik.

kurzgefasst: Zur Überwachung von Colitis-Patienten wird empfohlen, bei der jährlichen Koloskopie je 4 Kolonbiopsien im Quadranten in $10 \mathrm{~cm}$ Abständen aus allen Kolonabschnitten, d.h. insgesamt 40-50 Biopsien zu entnehmen.

\section{IEN und Therapiekonsequenz}

Bei eindeutiger, durch einen auswärtigen Referenzpathologen bestätigter hochgradiger intraepithelialer Neoplasie ist dem Patienten eine Proktokolektomie zu empfehlen (Abb.1). Beim Nachweis von niedriggradigen IEN und deren Bestätigung durch einen auswärtigen Referenzpathologen kann alternativ zur sofortigen Proktokolektomie mit dem Patienten eine Intensivierung der Überwachungsprogramms (kürzere Endoskopieintervalle von z.B. 3-6 Monaten, Chromo- bzw. Zoom-Endoskopie) und die Proktokolektomie erst beim Nachweis von hochgradigen IEN vereinbart werden (9). Diese Empfehlung der Expertenkommission (Konsensus-Konferenz CU, Berlin 2004) berücksichtigt neben früheren Studien und einer zusammenfassenden Analyse von Bernstein (1) v.a. die Arbeit von Lim et al. (9), in der das Risiko einer histopathologischen Progression bei initial diagnostizierter niedriggradiger IEN bei 10\% (nach 10 Jahren) lag.)

Bei der Klassifikation „fragliche IEN“ liegt eine besonders schlechte Übereinstimmung der histologischen Beurteilung zwischen verschiedenen Pathologen vor. Eine Analyse von 10 prospektiven Studien hat allerdings gezeigt, dass "fragliche IEN“ bei $28 \%$ der Patienten eine Progression zur hochgradigen IEN und bei 9\% der Patienten zum Karzinom aufweisen (1). Daher wird bei Vorliegen von fraglichen IEN eine endoskopische Kontrolle nach Intensivierung der antiinflammatorischen Therapie innerhalb von 3-6 Monaten empfohlen. Bestätigt sich dann eine niedriggradige IEN, kann alternativ zu weiteren intensivierten Kontrollen die Proktokolektomie empfohlen werden. Bei Vorliegen eines ausgeprägten Pseudopolypenrasens kann auch ohne Nachweis von IEN die Empfehlung zur Proktokolektomie sinnvoll sein, da ein solcher Darm nicht überwacht werden kann.

\section{DALM versus sporadische adenomatöse Polypen}

Sporadische adenomatöse Polypen treten bei CED-Patienten genauso häufig auf wie in der Normalbevölkerung. Die Unterscheidung der sporadischen adenomatösen Polypen von der 


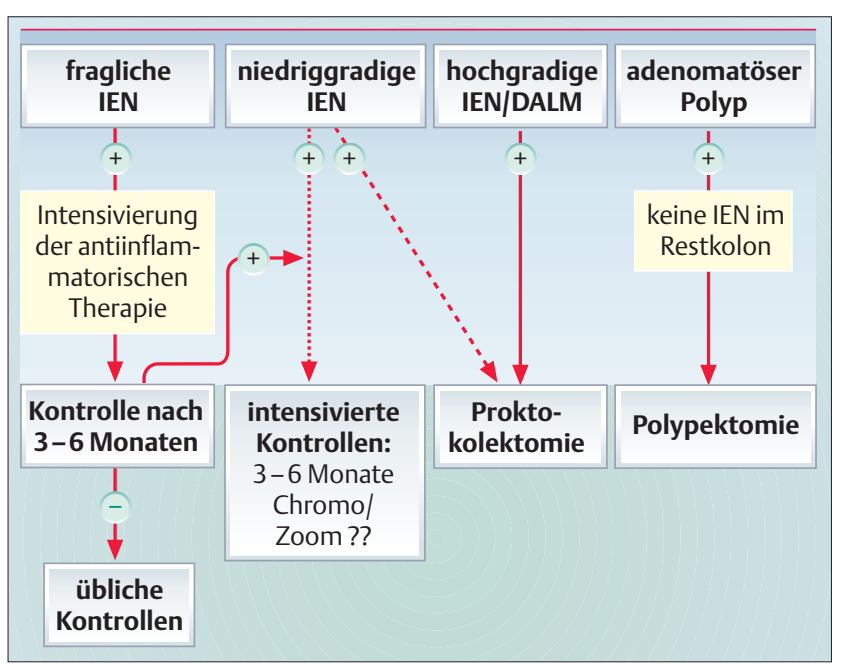

Abb.1: Karzinomprophylaxe bei Colitis ulcerosa: Konsequenzen bei Nachweis einer intraepithelialen Neoplasie. IEN=intraepitheliale Neoplasie in flacher Mukosa. DALM (dysplasia-associated lesion or mass) = intraepitheliale Neoplasie in erhabener Läsion, die in entzündeter Mukosa gelegen oder davon umgeben ist.

Dysplasia Associated Lesion or Mass (DALM), die sich auf endoskopisch-makroskopische und histologische Kriterien stützt, hat für den Colitis-Patienten wesentliche therapeutische Konsequenzen. Es liegen zwei Studien mit größerer Fallzahl vor $(5,12)$, in denen nach alleiniger Polypektomie von sporadischen adenomatösen Polypen bei CU über eine Nachbeobachtungszeit von 3,5 bzw. 4 Jahren erneut Adenome, jedoch keine Karzinome oder Schleimhautareale mit IEN nachgewiesen wurden. Bei Nachweis von IEN in der benachbarten Schleimhaut liegt jedoch ein erhöhtes Karzinomrisiko vor. Die KonsensusKonferenz CU (Berlin 2004) empfiehlt daher bei Nachweis eines sporadischen adenomatösen Kolonpolypen bei CU-Patienten die alleinige Polypektomie, wenn im Restkolon durch multiple Stufenbiopsien keine IEN nachgewiesen werden.

\section{kurzgefasst: Bei hochgradiger intraepithelialer Neoplasie (IEN) ist eine Proktokolektomie zu empfehlen, bei niedrig- gradigen IEN kann nach Einschätzung der individuellen Ri- siken eine Kontrollkoloskopie innerhalb von 3-6 Monaten durchgeführt werden. Adenomatöse Polypen in nicht-dys- plastischer umgebender Schleimhaut können auch bei CED-Patienten endoskopisch abgetragen werden.}

\section{Primärprävention des KRIK}

Retrospektive Fall-Kontroll-Studien wiesen auf den Nutzen der anti-entzündlichen Therapie mit 5-Aminosalicylaten (5-ASA) und Sulfasalazin in der Primärprävention desKRK bei CU hin. Dabei wurde eine Senkung des Karzinomrisikos durch eine regelmäßige 5-ASA-Einnahme (mindestens 1,2 g/d) bis zu 75\% gezeigt. Die Bedeutung der Entzündungsaktivität für das Entartungsrisiko wird dennoch kontrovers diskutiert. Eine Fortführung der Aminosalicylattherapie zur Karzinomprophylaxe sollte mit dem Patienten individuell anhand vorliegender Risikofaktoren besprochen werden. Sie ersetzt nicht die regelmäßige endoskopische Überwachung. Bei gleichzeitig bestehender primär sklerosierender Cholangitis wird empfohlen, Ursodeoxychol- säure einzusetzen. In einer plazebokontrollierten Studie konnte bei diesen Patienten das Darmkrebsrisiko dadurch um $74 \%$ gesenkt werden (11).

\section{Fazit}

Patienten mit langjähriger Colitis ulcerosa, und wahrscheinlich auch mit Crohn-Colitis, haben ein erhöhtes Risiko für das Auftreten eines KRK und sollten daher nach langjährigem Verlauf regelmäßig koloskopisch-bioptisch überwacht werden. Bei Nachweis von niedriggradigen IEN kann alternativ zur Proktokolektomie eine Intensivierung des Überwachungsprogramms in Form einer Kontrollendoskopie in 3-6 Monaten erwogen werden. Bei einer durch einen Referenzpathologen bestätigten hochgradigen IEN oder DALM sollte dem Patienten eine Proktokolektomie empfohlen werden. Ein adenomatöser Polyp in einem nicht dysplastischen Kolon kann analog zur Situation bei Nicht-CED-Patienten polypektomiert werden. Die konsequente Einnahme von Sulfasalazin bzw. 5-ASA-Präparaten kann das Karzinomrisiko senken.

\section{Literatur}

Bernstein CN, Shanahan F, Weinstein WM. Are we telling patients the truth about surveillance colonoscopy in ulcerative colitis? Lancet 1994; 343: 7174

2 Choi PM, Zelig MP. Similarity of colorectal cancer in Crohn's disease and ulcerative colitis: implications for carcinogenesis and prevention. Gut 1994; 35: 950-954

3 Eaden JA, Abrams KR, Mayberry JF. The risk of colorectal cancer in ulcerative colitis: a meta-analysis. Gut 2001; 48: 526-535

4 Ekbom A, Helmick C, Zack M, Adami HO. Increased risk of large-bowel cancer in Crohn's disease with colonic involvement. Lancet 1990; 336: 357359

5 Engelsgjerd M, Farraye FA, Odze RD. Polypectomy may be adequate treatment for adenoma-like dysplastic lesions in chronic ulcerative colitis. Gastroenterology 1999; 117: 1288-1294; discussion 1488-1491.

6 Jess T, Winther KV, Munkholm P, Langholz E, Binder V. Intestinal and extraintestinal cancer in Crohn's disease: follow-up of a population-based cohort in Copenhagen County, Denmark. Aliment Pharmacol Ther 2004; 19: 287-293

7 Kiesslich R, Fritsch J, Holtmann M, Koehler H, Stolte M, Kanzler S, Nafe B, Jung M, Galle P, Neurath MF. Methylene blue-aided chromoendoscopy for the detection of intraepithelial neoplasia and colon cancer in ulcerative colitis. Gastroenterology 2003; 124: 880-888

8 Lashner BA, Watson AJM. Colorectal cancer in ulcerative colitis: surveillance. London: BMJ Books, In: McDonal JWD, Burroughs AK, Feagan BG, editors. Evidence based gastroenterology and hepatology. 1999: 221-229

9 Lim CH, Dixon MF, Vail A, Forman D, Lynch DA, Axon AT. Ten year follow up of ulcerative colitis patients with and without low grade dysplasia. Gut 2003; 52: 1127-1132

10 Mpofu C, Watson A, Rhodes J. Strategies for detecting colon cancer and/or dysplasia in patients with inflammatory bowel disease. Cochrane Database Syst Rev 2004; 2: CD000279

11 Pardi DS, Jr. Loftus EV, Kremers WK, Keach J, Lindor KD. Ursodeoxycholic acid as a chemopreventive agent in patients with ulcerative colitis and primary sclerosing cholangitis. Gastroenterology 2003; 124: 889-893

12 Rubin PH, Friedman S, Harpaz N, Goldstein E, Weiser J, Schiller J et al. Colonoscopic polypectomy in chronic colitis: conservative management after endoscopic resection of dysplastic polyps. Gastroenterology 1999; 117: 1295-1300

13 Taylor BA, Pemberton JH, Carpenter HA, Levin KE, Schroeder KW, Welling DR et al. Dysplasia in chronic ulcerative colitis: implications for colonoscopic surveillance. Dis Colon Rectum 1992; 35: 950-956

14 Winther KV, Jess T, Langholz E, Munkholm P. Binder Survival and causespecific mortality in ulcerative colitis: follow-up of a population-based cohort in Copenhagen County. Gastroenterology 2003; 125: 1576-1582

15 Hoffmann JC, Zeitz M, Bischoff S, Brambs H, Bruch H, Buhr H, Dignaß A, Fleig W, Fölsch U, Höhne W, Jantschek G, Kaltz B, Keller K, Knebel U, Kroesen A Kruis W, Matthes H, Moser G, Mundt S, Pox C, Reinshagen M, Reißmann A Riemann J, Rogler G, Schmiegel W, Schölmerich J, Schreiber S, Schwandner O, Selbmann H, Stange E, Utzig M, Wittekind C. Diagnostik und Therapie der Colitis ulcerosa: Ergebnisse einer evidenzbasierten Konsensuskonferenz der Deutschen Gesellschaft für Verdauungs- und Stoffwechselerkrankungen zusammen mit dem Kompetenznetz chronisch entzündliche Darmerkrankungen. Z Gastroenterologie, im Druck 